\title{
Analysis of a Segment Type Switched Reluctance Generator for Wind Power Generation
}

\author{
Takashi Iwanaga, Sho Fukumoto, Tsuyoshi Higuchi, Yuichi Yokoi, Takashi Abe \\ Nagasaki University \\ Department of Electrical and Electronic Engineering \\ Nagasaki city, Japan \\ bb52111204@cc.nagasaki-u.ac.jp
}

\begin{abstract}
The authors proposed a novel segment type reluctance generator (RG) and presented the experimental performance. It had similar induced voltage to the usual three phase synchronous generator, but the efficiency was low. This paper presents a novel segment type switched reluctance generator (SRG) to improve the efficiency and describes its FEM analysis for wind power generation. The performance is compared with the segment type RG.
\end{abstract}

Keywords-component; Switched reluctance generator, Wind power generation, Rare earth less machine

\section{INTRODUCTION}

Recently, permanent magnet synchronous generator has been developed for bulk wind power generation because of high efficiency [1].

The authors previously proposed a novel segment type reluctance generator (RG) to decrease the cost, and presented the experimental performances. It was composed of a stator with an exciting winding and 3-phases full-pitch armature windings and a rotor with segment cores embedded in an aluminum block. It produced similar induced voltage to the usual 3-phases synchronous generator. It had special merits of low cost, rare earth less, robust structure, but the power factor and efficiency was low.

This paper proposes a novel segment type switched reluctance generator (SRG) to improve the efficiency and describes its design analysis results for $2 \mathrm{MW}$ and direct drive type generator. The performance is compared with the segment type $\mathrm{RG}[2]$.

\section{SEGMENT TYPE RG AND SRG}

\section{A. Construction and characteristics}

Fig. 1 shows model of the segment type RG and SRG. The number of stator poles is 6 and the number of rotor cores is 4 . The stator has full-pitch armature winding and has a series connected excitation winding for $R G$ and three phases excitation windings for SRG. The rotor is composed of an aluminum block and segment cores for both generators.
In $R G$, a direct current is given to the excitation winding at all times. The electromotive force is induced on the armature windings in response to time variation of the flux.

In SRG, the three phases exciting currents are switched according to the rotational angles shown in Fig. 2. Fig.3 shows flux distribution at aligned position. When the rotor rotates in a counterclockwise direction from the initial position of Fig. 1 and the rotational angle becomes $30 \mathrm{deg}$, inductance of the phase-A winding reaches the maximum value. The inductance decreases with the rotor rotating more. If the phase-A winding is excited by direct current pulse from 30 degree to 60 degree like Fig. 2, braking torque is generated and the electromotive force is induced on the armature winding.

Fig. 4 shows the external load circuit. Induced voltage is rectified by the full wave rectifier circuit.

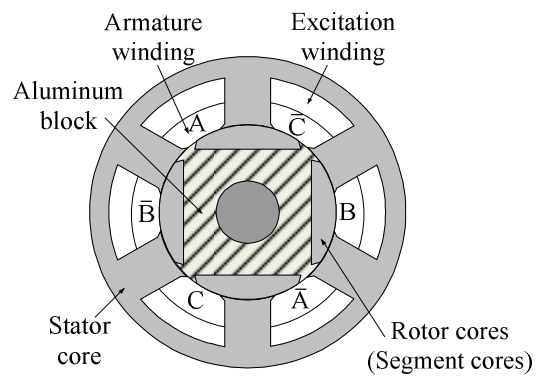

Figure 1. Construction of segment type RG and SRG

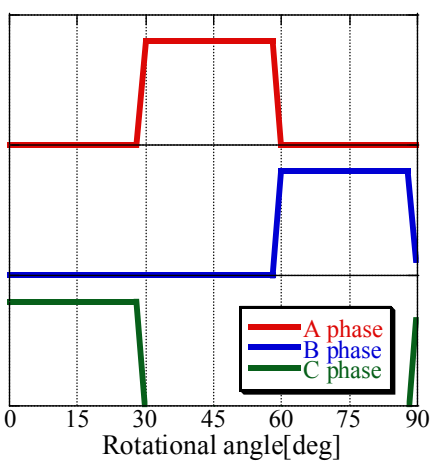

Figure 2. Input current 


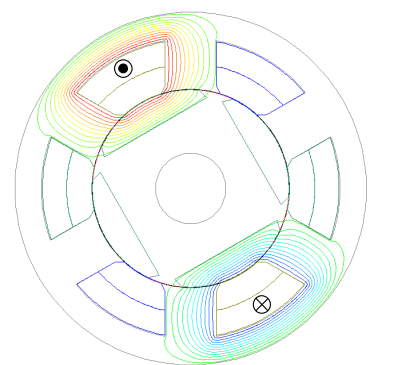

Figure 3. Magnetic flux on the aligned position (angle: 30deg)

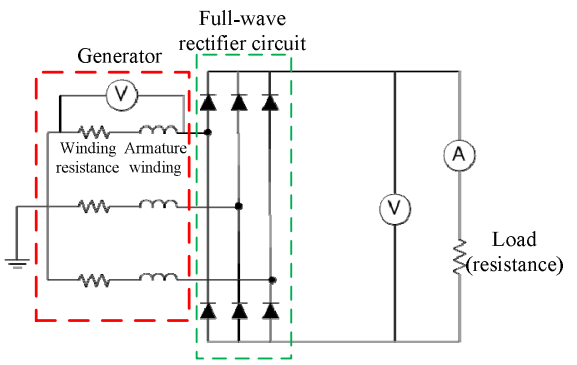

Figure 4. External circuit (load side) block, iron loss, copper loss, mechanical loss, stray load loss.

$$
\eta=\frac{P}{P+P_{e}+P_{i}+P_{c}+P_{s}+P_{m}} \times 100[\%]
$$

$P$ : Output $P_{e}$ : Eddy current loss $P_{i}$ : Iron loss $P_{c}$ : Copper loss $P_{s}$ : Stray load loss $P_{m}$ : Mechanical loss

Table I shows calculated loss and efficiency. The copper loss of the SRG is smaller than the RG. Stray load loss $P_{s}$ is estimated to $30 \%$ of the copper loss $P_{c}$.

The iron loss of both generators is small but the eddy current loss developed in the aluminum block is very high. The iron loss of the RG is $46.89 \mathrm{~kW}$ and that of the $\mathrm{SRG}$ is $16.69 \mathrm{~kW}$. It is decreased by $64 \%$ compared with the RG. It will be caused by the difference of the magnetic path.

The eddy current loss is calculated by FEM. The eddy current loss of the RG is $884 \mathrm{~kW}$ and that of the SRG is $470 \mathrm{~kW}$. It is decreased by $47 \%$ compared with the RG. It will be caused by that the linkage flux to an aluminum block is decreased.

The efficiency of the SRG is $73.7 \%$ and that of the RG is $41.9 \%$.

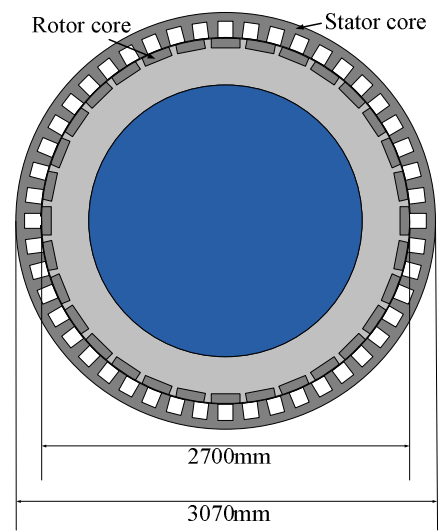

Figure 5. SRG full model (stator: 48 poles) SRG. The maximum output of the RG is $1.1 \mathrm{MW}$ and the SRG is $2.3 \mathrm{MW}$. The output of the RG is small because the RG has motor torque which prevents generation as well as braking torque. The output of the SRG is increased by $105 \%$ compared with the RG.

\section{Characteristics of the eddy current loss}

Fig. 8 shows the characteristics of the eddy current loss. When the load current is small, the eddy current loss of the SRG is nearly as same as that of the RG. However, when the load current is large, the loss of the RG is larger than that of the SRG. It will be caused by the difference of the flux between the $\mathrm{RG}$ and the SRG. The effect of the flux leakage is enormous because the excitation current always flow through all excitation windings.

\section{E. Efficiency characteristics}

We calculate efficiency using Equation (1). We consider following losses; eddy current loss on the aluminum rotor 


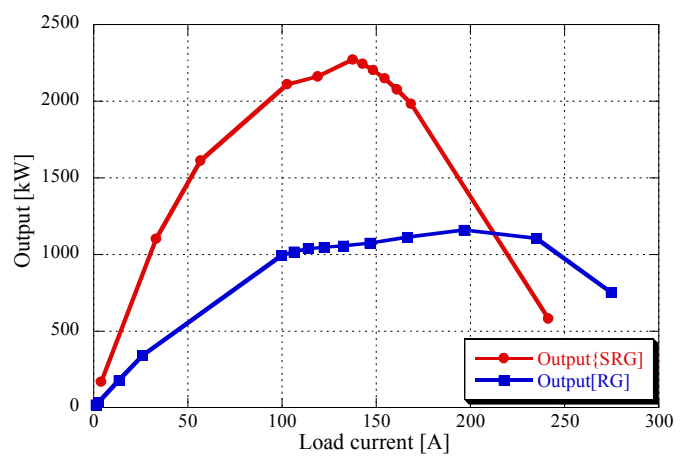

Figure 7. Output characteristics

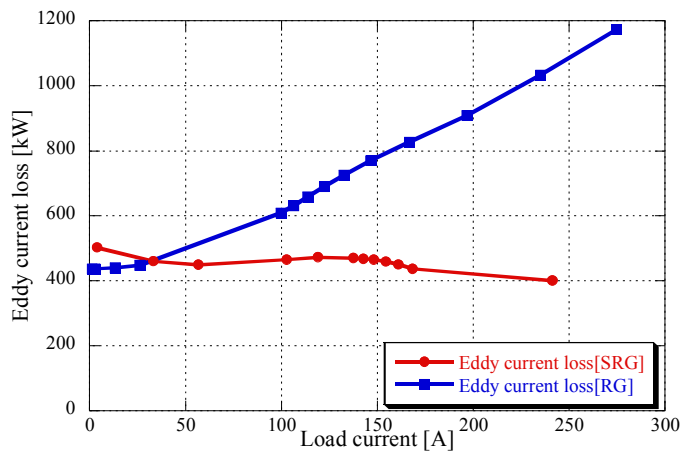

Figure 8. Characteristics of the eddy current loss

TABLE I. LOSS AND EFFICIENCY (MAXIMUM OUTPUT)

\begin{tabular}{|c|c|c|}
\hline model & RG & SRG \\
\hline Maximum output [kW] & 1110 & 2274 \\
\hline Copper loss(excitation)[kW] & 301.2 & 123.6 \\
\hline Copper loss(armature) [kW] & 166.3 & 125.6 \\
\hline Stray load loss [kW] & 140.3 & 74.77 \\
\hline Mechanical loss [kW] & 0.489 & 0.489 \\
\hline Iron loss [kW] & 46.89 & 16.69 \\
\hline Eddy current loss [kW] & 884 & 470 \\
\hline Efficiency [\%] & 41.89 & 73.71 \\
\hline
\end{tabular}

\section{DESIGN FOR REDUCTION OF THE EDDY CURRENT LOSS}

In this section, we designed the SRG for reduction of the eddy current loss. Specifically, we examined the height of the aluminum block because the eddy current loss developed in the aluminum block is very high.

Fig.9 shows the definition of the aluminum height. The aluminum block is divided into four. We lowered the height of the aluminum block every height and analyzed each model about output and eddy current loss characteristics.

\section{A. Output characteristics}

Fig.10 shows the output characteristics after modification of the aluminum height. As the aluminum height is lowered, the output becomes larger. The reason is the difference of maximum inductance and minimum inductance becomes more extreme. The leakage flux is decreased by reduction of the aluminum.

The maximum output of the $0 / 4$ model is about $3 \mathrm{MW}$. It is increased by $30 \%$ compared with the $4 / 4$ model. As a result, we can expect an enlargement of output by reduction of the aluminum.

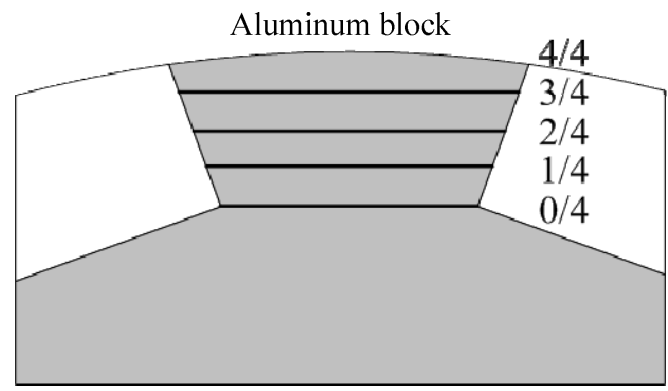

Figure 9. Definition of the aluminum height

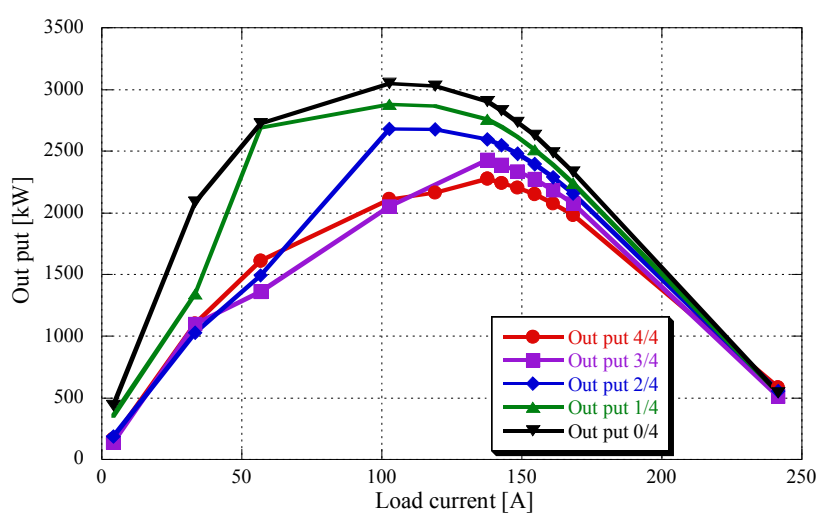

Figure 10. Output characteristics

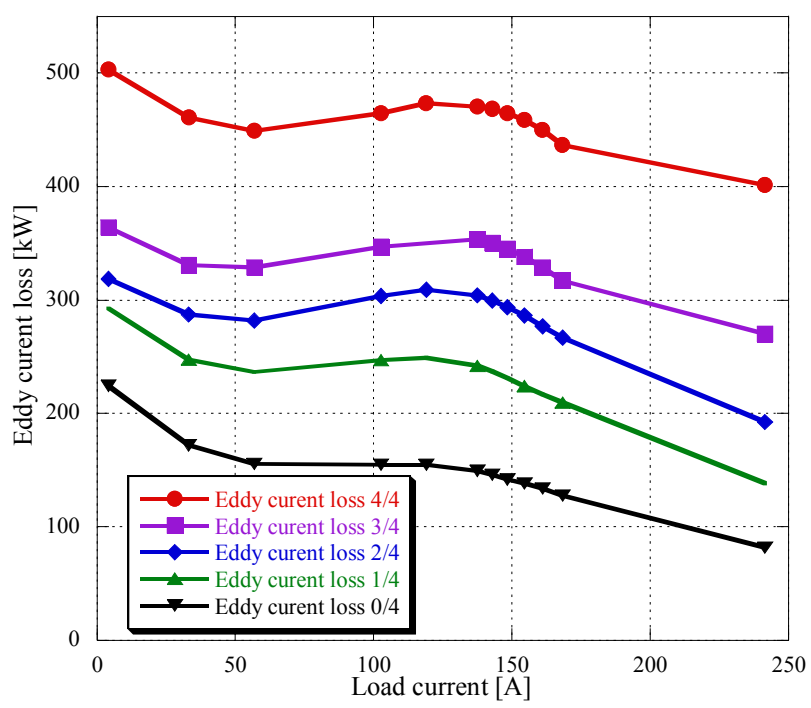

Figure 11. Characteristics of the eddy current loss 


\section{B. Characteristics of the eddy current loss}

Fig. 11 shows characteristics of the eddy current loss. As the aluminum height is lowered, the eddy current loss decreased on the whole. It will be caused by reduction of the leakage flux through the aluminum block. The eddy current loss of the $0 / 4$ model is decreased by about $50 \%$ compared with the $4 / 4$ model.

\section{Efficiency characteristics}

Fig.12 shows the efficiency characteristics. The maximum efficiency is $78.8 \%$ in $0 / 4$ model.

\section{CONCLUSION}

In this paper, we designed and analyzed the $2 \mathrm{MW}$ class segment type reluctance generator and the switched reluctance generator. The maximum output of the RG was $1.1 \mathrm{MW}$ and the SRG was $2.3 \mathrm{MW}$. The efficiency of the RG was $41.9 \%$ and the SRG was $73.7 \%$. Also, the eddy current loss which developed on an aluminum block is decreased and the output is enlarged by reduction of the aluminum.

Next time, we will show the experimental performances of the $2 \mathrm{~kW}$ segment type RG and SRG in Fig. 12.

\section{ACKNOWLEDGMENT}

This work was supported by JSPS KAKENHI Grant number 22360119.

\section{REFERENCES}

[1] T. Kuroiwa, K. Karikomi, Y. Hayashi, M. Shibata, and Y. Ueda: "New Products and Technologies of Mitsubishi Wind Turbines", Mitsubishi Heavy Industry Technical Report, Vol. 41, No. 3, pp. 170-173 (2004-5)

[2] K. Yamada, S. Fukumoto, T. Higuchi: "The Fundamental Characteristics and principle of Novel Switched Reluctance Generator", Journal of conference of Industrial applications Society, Vol.3, No.3-40, pp.271$272(2010.8)$

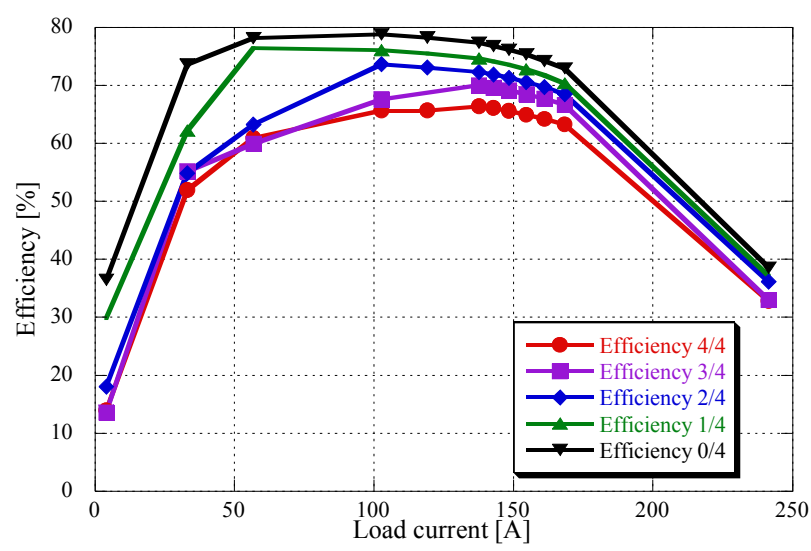

Figure 12. Efficiency characteristics

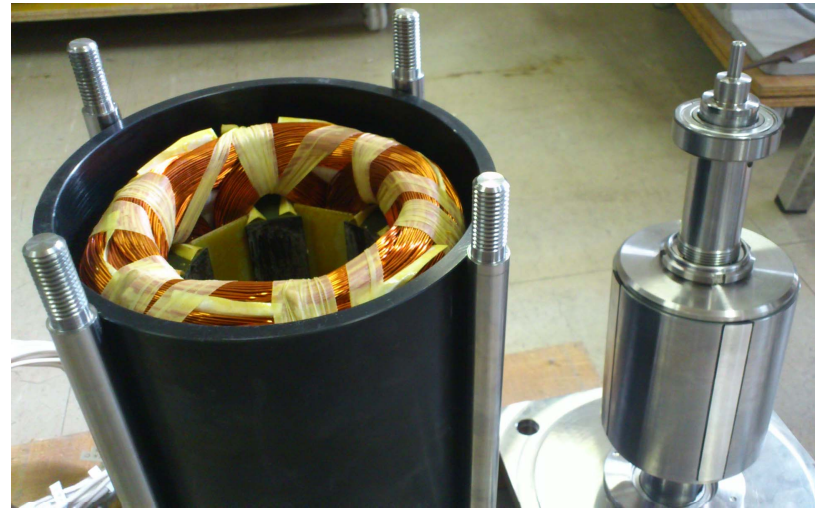

Figure 12. The $2 \mathrm{~kW}$ segment type SRG 